MATHEMATICS OF COMPUTATION

Volume 68, Number 227, Pages 1299-1301

S 0025-5718(99)01095-9

Article electronically published on February 19, 1999

\title{
SQUARE PRODUCT OF THREE INTEGERS IN SHORT INTERVALS
}

\author{
L. HAJDU AND Á. PINTÉR
}

\begin{abstract}
In this paper we list all the integer triplets taken from an interval
\end{abstract} of length $\leq 12$, whose products are perfect squares.

\section{INTRODUCTION}

Let $f$ and $k$ be positive integers with $f \leq k$. The sets of distinct integers $n_{1}, \ldots, n_{f} \in[n+1, \ldots, n+k]$ with the property that there is a nontrivial way to multiply them to obtain a perfect power was investigated by Erdős and Turk [ET]. This question is related to the Erdős-Selfridge theorem (see [ES]), which states that the product of two or more consecutive integers is never a perfect power - that is, if $f=k \geq 2$, then the equation

$$
\prod_{i=1}^{f} n_{i}=x^{m} \quad(x \in \mathbb{N}, m \geq 2)
$$

has no solutions. Moreover, Erdős and Turk conjectured (cf. [ET]) that (1) has no solutions with $(k, f, m)=(4,3,2)$. This conjecture was verified by Tzanakis [T].

In this paper we list all the integer triplets $(f=3)$ taken from a short interval $(k \leq 12)$ whose products are perfect squares.

\section{RESUlT}

Now we formulate our result.

Theorem. Let $(a, b, c) \in \mathbb{Z}^{3}$ with $a<b<c$ such that $c-a=k-1<12$. If $a b c \neq 0$ is a perfect square, then the triplet $(a, b, c)$ is one of the following:

$k=5:(-2,-1,2),(2,3,6)$;

$k=6:(-4,-1,1),(3,6,8),(5,8,10),(240,243,245)$;

$k=7:(-4,-2,2),(-3,-1,3),(2,4,8),(6,8,12),(48,50,54)$;

$k=8:(-4,-3,3),(1,2,8),(2,8,9),(7,8,14),(21,27,28)$;

$k=9:(-6,-3,2),(-4,-1,4),(1,4,9),(2,5,10),(12,15,20),(24,27,32)$, $(242,245,250)$;

Received by the editor February 19, 1998.

1991 Mathematics Subject Classification. Primary 11D25.

The first author's research was supported in part by the Hungarian Academy of Sciences, by Grants 014245 and T 016975 from the Hungarian National Foundation for Scientific Research and by the Pro Regione Foundation of the Hajdúsági Agráripari RT.

The second author's research was supported in part by the Hungarian Academy of Sciences and by Grants 16975 and 19479 from the Hungarian National Foundation for Scientific Research.

(C)1999 American Mathematical Society 


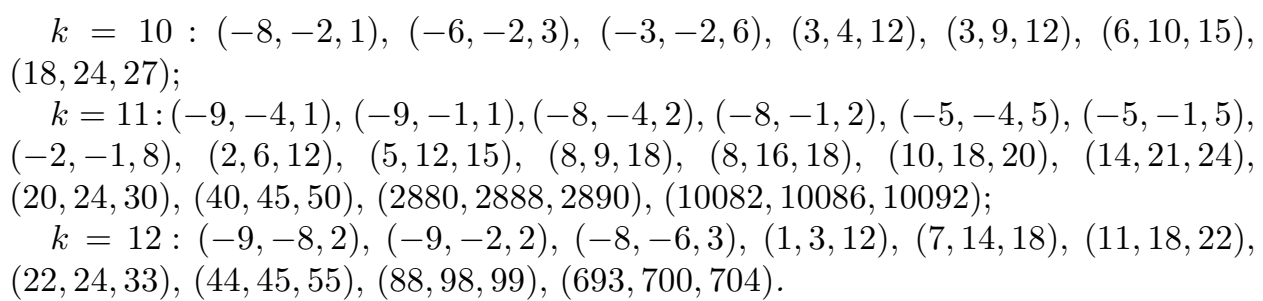

As a consequence of the theorem we obtain that the interval $[44,45, \ldots, 55]$ is the smallest one which contains two disjoint triplets of positive integers with the relevant property: $\{44,45,55\}$ and $\{48,50,54\}$.

\section{Proof}

To prove our theorem, we will reduce equation (1) to several elliptic equations. Recently, Gebel, Pethő and Zimmer [GPZ], and independently Stroeker and Tzanakis $[\mathrm{ST}]$, have developed an algorithm for solving elliptic equations. Their method is based on the approach of Zagier [Z], and on the recent estimates of linear forms in elliptic logarithms due to David [D]. The algorithm outlined in [GPZ] has been implemented by Gebel in the program package SIMATH (cf. [SIM]), and we use this program package to solve our elliptic equations.

Proof of the Theorem. Let $(a, b, c) \in \mathbb{Z}^{3}$ be a triplet with the desired property, and put $x=a, u=b-a$ and $v=c-a$. To prove the theorem we have to solve the system of elliptic equations

$$
x(x-u)(x-v)=y^{2}
$$

with $0<u<v<12$ in integers $x, y$. Using the results of Erdős and Selfridge [ES], and Tzanakis [T], we may suppose that $v \geq 4$, and we obtain 52 equations. By a simple substitution we transform these elliptic equations into Weierstrass normal form, and we can solve them by SIMATH. We obtained just the solutions listed in our theorem.

\section{ACKNOWLEDGMENTS}

We are grateful to the referee for his useful remarks and observations.

\section{REFERENCES}

[D] S. David, Minorations de formes linéaires de logarithmes elliptiques, Mémoires de la Société Mathématique de France, Nouvelle série, Supplément au Bulletin de la Société Mathématique de France No 62 (1995). MR 98f:11078

[ES] P. Erdős and J. L. Selfridge, The product of consecutive integers is never a power, Illinois J. Math. 19 (1975), 292-301. MR 51:12692

[ET] P. Erdős and J. Turk, Products of integers in short intervals, Acta Arith. 44 (1984), 147-174. MR 86d:11073

[GPZ] J. Gebel, A. Pethő and H. G. Zimmer, Computing integral points on elliptic curves, Acta Arith. 68 (1994), 171-192. MR 95i: 11020

[SIM] SIMATH, Saarbrücken, 1993.

[ST] R. J. Stroeker and N. Tzanakis, Solving elliptic diophantine equations by estimating linear forms in elliptic logarithms, Acta Arith. 67 (1994), 177-196. MR 95m:11056 
[T] N. Tzanakis, On the diophantine equation $x^{2}-D y^{4}=k$, Acta Arith. 46 (1986), 257-269. MR 87k:11031

[Z] D. Zagier, Large integral points on elliptic curves, Math. Comp. 48 (1987), 425-436. MR 87k:11062

Institute of Mathematics and Informatics, Lajos Kossuth University, H-4010 DebreCen, Pf. 12, Hungary

E-mail address: hajdul@math.klte.hu

E-mail address: apinter@math.klte.hu 